ORIGINAL ARTICLE

\section{Combined BRAF and MEK Inhibition versus BRAF Inhibition Alone in Melanoma}

\author{
G.V. Long, D. Stroyakovskiy, H. Gogas, E. Levchenko, F. de Braud, J. Larkin \\ C. Garbe, T. Jouary, A. Hauschild, J.J. Grob, V. Chiarion Sileni, C. Lebbe, \\ M. Mandalà, M. Millward, A. Arance, I. Bondarenko, J.B.A.G. Haanen, \\ J. Hansson, J. Utikal, V. Ferraresi, N. Kovalenko, P. Mohr, V. Probachai, \\ D. Schadendorf, P. Nathan, C. Robert, A. Ribas, D.J. DeMarini, J.G. Irani, \\ M. Casey, D. Ouellet, A.-M. Martin, N. Le, K. Patel, and K. Flaherty
}

A BSTRACT

\section{BACKGROUND}

Combined BRAF and MEK inhibition, as compared with BRAF inhibition alone, delays the emergence of resistance and reduces toxic effects in patients who have melanoma with BRAF V600E or V600K mutations.

\section{METHODS}

In this phase 3 trial, we randomly assigned 423 previously untreated patients who had unresectable stage IIIC or stage IV melanoma with a BRAF V600E or V600K mutation to receive a combination of dabrafenib (150 mg orally twice daily) and trametinib ( $2 \mathrm{mg}$ orally once daily) or dabrafenib and placebo. The primary end point was progression-free survival. Secondary end points included overall survival, response rate, response duration, and safety. A preplanned interim overall survival analysis was conducted.

\section{RESULTS}

The median progression-free survival was 9.3 months in the dabrafenib-trametinib group and 8.8 months in the dabrafenib-only group (hazard ratio for progression or death in the dabrafenib-trametinib group, 0.75 ; $95 \%$ confidence interval [CI], 0.57 to $0.99 ; \mathrm{P}=0.03$ ). The overall response rate was $67 \%$ in the dabrafenib-trametinib group and $51 \%$ in the dabrafenib-only group $(\mathrm{P}=0.002)$. At 6 months, the interim overall survival rate was $93 \%$ with dabrafenib-trametinib and $85 \%$ with dabrafenib alone (hazard ratio for death, 0.63 ; $95 \%$ CI, 0.42 to 0.94 ; $\mathrm{P}=0.02$ ). However, a specified efficacy-stopping boundary (two-sided $\mathrm{P}=0.00028$ ) was not crossed. Rates of adverse events were similar in the two groups, although more dose modifications occurred in the dabrafenib-trametinib group. The rate of cutaneous squamous-cell carcinoma was lower in the dabrafenib-trametinib group than in the dabrafenib-only group ( $2 \%$ vs. $9 \%$ ), whereas pyrexia occurred in more patients (51\% vs. $28 \%$ ) and was more often severe (grade $3,6 \%$ vs. $2 \%$ ) in the dabrafenib-trametinib group.

\section{CONCLUSIONS}

A combination of dabrafenib and trametinib, as compared with dabrafenib alone, improved the rate of progression-free survival in previously untreated patients who had metastatic melanoma with BRAF V600E or V600K mutations. (Funded by GlaxoSmithKline; Clinical Trials.gov number, NCT01584648.)
The authors' full names, academic degrees, and affiliations are listed in the Appendix. Address reprint requests to Dr. Long at the Melanoma Institute Australia, University of Sydney, and the Mater Hospital, 40 Rocklands Rd., North Sydney, NSW 2060, Australia, or at georgina.long@ sydney.edu.au.

This article was published on September 29, 2014, at NEJM.org.

N Engl J Med 2014;371:1877-88. DOI: 10.1056/NEJMoal406037 Copyright (๑) 2014 Massachusetts Medical Society. 
T ARGETED INHIBITION OF THE RAF-MEKERK (MAPK) pathway with BRAF inhibitors dabrafenib or vemurafenib, as compared with chemotherapy, improves the progressionfree and overall survival of patients who have metastatic melanoma with BRAF V600 mutations. ${ }^{1,2}$ However, resistance develops in a majority of patients, resulting in a median progressionfree survival of 6 to 7 months. ${ }^{3,4}$ Most reported resistance mechanisms reactivate the MAPK pathway. ${ }^{5-7}$ In addition, BRAF-inhibitor-induced paradoxical activation of the MAPK pathways-10 can result in secondary cancers, including cutaneous squamous-cell carcinoma, and may reactivate RAS-mutant tumors. ${ }^{11-13}$ Independently, singleagent trametinib, a MEK inhibitor, improves the overall survival of patients with BRAF V600 mutation-positive metastatic melanoma, as compared with chemotherapy, and is not associated with paradoxical activation. ${ }^{14}$

The combination of BRAF and MEK inhibition, as compared with single-agent BRAF inhibition, delayed the emergence of resistance and decreased the incidence of cutaneous hyperproliferative lesions in preclinical models. ${ }^{12,15,16}$ This finding was confirmed in an unblinded, randomized, phase 2 study of a combination of dabrafenib and trametinib, as compared with dabrafenib alone, with crossover permitted at the time of disease progression for those receiving dabrafenib alone. ${ }^{17}$ The median progressionfree survival was 9.4 months with combination therapy versus 5.8 months with dabrafenib alone (hazard ratio for progression or death in the dabrafenib-trametinib group, 0.39 ; $95 \%$ confidence interval [CI], 0.25 to $0.62 ; \mathrm{P}<0.001$ ) and the corresponding response rate was $76 \%$ versus $54 \%(\mathrm{P}=0.03) .{ }^{17}$ The rate of cutaneous squamouscell carcinoma was lower in the dabrafenib-trametinib group than in the dabrafenib-only group (7\% vs. $19 \%)$.

We conducted a double-blind, randomized, phase 3 study without crossover to compare the combination of dabrafenib and trametinib with dabrafenib alone as first-line therapy in patients who had metastatic melanoma with BRAF V600E or V600K mutations.

METHODS

\section{PATIENTS}

Eligible patients had histologically confirmed, unresectable stage IIIC or stage IV metastatic melanoma with BRAF V600E or V600K mutations, as determined by means of an investigationaluse-only polymerase-chain-reaction assay (ThxID BRAF Assay, bioMérieux) performed at a central reference laboratory. Patients were not eligible if they had previous systemic anticancer therapy (including BRAF or MEK inhibitors). Patients with brain metastases that had been definitively treated and stable for at least 12 weeks were eligible to participate. Additional inclusion and exclusion criteria are provided in the Supplementary Appendix, available with the full text of this article at NEJM.org. Written informed consent was obtained from all study patients.

\section{STUDY DESIGN AND TREATMENTS}

From May 2012 through January 2013, we screened 947 patients at 113 centers worldwide. We randomly assigned 423 of these patients, in a 1:1 ratio, to receive either a combination of oral dabrafenib (150 mg twice daily) and oral trametinib (2 mg once daily) or oral dabrafenib (150 mg twice daily) and placebo. Patients were stratified according to the baseline lactate dehydrogenase level and BRAF genotype (Fig. S1 in the Supplementary Appendix).

\section{STUDY END POINTS}

The primary end point was investigator-assessed progression-free survival, defined as the time from randomization until radiologic disease progression or death from any cause. The secondary end points were overall survival, response rate, response duration, safety, and pharmacokinetics, as defined in the Supplementary Appendix. An independent central review committee whose members were unaware of study-group assignments reviewed radiologic findings on which a sensitivity analysis of progression-free survival was based. No interim analyses were performed for efficacy or futility with respect to the primary end point.

\section{STUDY ASSESSMENTS}

Tumor assessments were conducted according to Response Evaluation Criteria in Solid Tumors (RECIST), version $1.1{ }^{18}$ at baseline, at week 8 , every 8 weeks until week 56, and then every 12 weeks until disease progression, death, or withdrawal from the study. Patients who were eligible for continued treatment beyond progression underwent tumor assessments according to the protocol. (Eligibility criteria for this assessment 
are provided in the Supplementary Appendix.) All responses were confirmed on either computed tomography or magnetic resonance imaging, with scanning obtained at least 4 weeks after the first RECIST response. Adverse events were graded by the site investigator according to the National Cancer Institute Common Terminology Criteria for Adverse Events, version 4.0, ${ }^{19}$ throughout the study until 30 days after the discontinuation of study treatment.

\section{STUDY OVERSIGHT}

This study was funded by the sponsor, GlaxoSmithKline. The protocol was approved by the institutional review board or human research ethics committee at each participating center and com-

\begin{tabular}{|c|c|c|c|}
\hline Characteristic & $\begin{array}{l}\text { Dabrafenib } \\
\text { plus Trametinib } \\
(\mathrm{N}=211)\end{array}$ & $\begin{array}{c}\text { Dabrafenib } \\
\text { Alone } \\
(\mathrm{N}=212)\end{array}$ & $\begin{array}{l}\text { All Patients } \\
(\mathrm{N}=423)\end{array}$ \\
\hline Median age (range) $-\mathrm{yr}$ & $55.0(22-89)$ & $56.5(22-86)$ & $56.0(22-89)$ \\
\hline Male sex - no. (\%) & $111(53)$ & $114(54)$ & $225(53)$ \\
\hline Previous immunotherapy — no./total no. (\%) & $56 / 210(27)$ & $61 / 211(29)$ & $117 / 421(28)$ \\
\hline \multicolumn{4}{|l|}{ ECOG performance score - no./total no. (\%) $\dagger$} \\
\hline 0 & $155 / 210(74)$ & $150 / 211(71)$ & $305 / 421(72)$ \\
\hline 1 & $55 / 210(26)$ & $61 / 211(29)$ & $116 / 421(28)$ \\
\hline \multicolumn{4}{|l|}{$B R A F$ mutation - no. (\%) } \\
\hline V600E & $179(85)$ & $181(85)$ & $360(85)$ \\
\hline V600K & $32(15)$ & $30(14)$ & $62(15)$ \\
\hline \multicolumn{4}{|l|}{ Tumor stage - no. (\%)』 } \\
\hline IVMlc & $142(67)$ & $138(65)$ & $280(66)$ \\
\hline IIIc, IVMla, or IVMlb & $69(33)$ & $73(34)$ & $142(34)$ \\
\hline \multicolumn{4}{|l|}{ Metastasis stage - no. (\%) } \\
\hline M0 & $5(2)$ & $10(5)$ & $15(4)$ \\
\hline $\mathrm{Mla}$ & $19(9)$ & $31(15)$ & $50(12)$ \\
\hline Mlb & $45(21)$ & $32(15)$ & $77(18)$ \\
\hline Mlc & $142(67)$ & $138(65)$ & $280(66)$ \\
\hline \multicolumn{4}{|l|}{ Lactate dehydrogenase level — no./total no. (\%) } \\
\hline$>U L N$ & $77 / 210(37)$ & $71 / 211(34)$ & $148 / 421(35)$ \\
\hline$\leq \mathrm{ULN}$ & $133 / 210(63)$ & $140 / 211(66)$ & $273 / 421(65)$ \\
\hline \multicolumn{4}{|l|}{ Visceral disease - no. (\%) } \\
\hline Yes & $165(78)$ & $145(68)$ & $310(73)$ \\
\hline No & $46(22)$ & $66(31)$ & $112(26)$ \\
\hline \multicolumn{4}{|l|}{ Number of disease sites — no./total no. (\%)\| } \\
\hline$\leq 2$ & $109 / 210(52)$ & $119 / 211(56)$ & $228 / 421(54)$ \\
\hline$\geq 3$ & $101 / 210(48)$ & $92 / 211(44)$ & $193 / 421(46)$ \\
\hline
\end{tabular}

* In the dabrafenib-trametinib group, 1 patient underwent randomization in error, and no baseline data were recorded for this patient in four of the listed categories (total number of patients in these categories, 210). In the dabrafenib-only group, 1 patient underwent randomization in error, and no baseline data were entered for this patient in any listed category (total number of patients in all categories, 211). There were no significant differences between the groups. ULN denotes upper limit of the normal range.

$\dagger$ Eastern Cooperative Oncology Group (ECOG) performance status ranges from 0 to 5, with higher numbers indicating increasing impairment in activities of daily living.

$\checkmark$ A patient with both BRAF V600E and V600K mutations was included in the V600K subgroup.

$\int$ The extent of metastatic melanoma is described as the American Joint Committee on Cancer stage.

I Visceral disease was defined as metastasis to the soft internal organs, including the lungs, heart, and the organs of the digestive, excretory, reproductive, and circulatory systems but excluding lymph nodes.

$\|$ This category refers to the number of unique target and nontarget sites that were identified by the investigator on the basis of Response Evaluation Criteria in Solid Tumors (RECIST) rather than the number of metastases. 
plied with country-specific regulatory requirements. The study was conducted in accordance with the provisions of the Declaration of Helsinki and Good Clinical Practice guidelines. The first and last authors and representatives of the sponsor designed the study. Data collection was performed by staff members employed at each study site and was monitored by the sponsor. The first author wrote the initial drafts of the manuscript, with support from the last author and representatives of the sponsor. All authors and sponsor representatives had full access to the study data and were involved in the data analysis. All the authors made the decision to submit the manuscript for publication and vouch for the accuracy of the data and the fidelity of the study to the protocol, which is available at NEJM.org. No one who is not listed as an author contributed to the writing of the manuscript.

\section{STATISTICAL ANALYSIS}

This report is based on data as of August 2013, when the prespecified number of disease progressions or deaths (whichever came first) had occurred. The study was initially designed with a power of more than $90 \%$ to detect a $41 \%$ reduction in the risk of disease progression or death (hazard ratio, 0.59) in the dabrafenib-trametinib group, as compared with the dabrafenib-only group, at a one-sided type I error rate of 0.025 . The study was overenrolled by approximately $24 \%$ (423 actual enrollees vs. the target of 340 ). To increase the precision of the median progression-free survival estimate in the dabrafenib-trametinib group, the final analysis was planned after 193 events had occurred, which maintained the same ratio of events to patients as originally planned. As a result, the power increased from $90 \%$ to $95 \%$. A prespecified interim analysis of overall survival was planned at the time of the analysis of progression-free survival. The stopping boundary for the interim analysis of overall survival was a two-sided alpha level of less than 0.00028 . A final overall survival analysis will be conducted when $70 \%$ of the patients who underwent randomization have died or been lost to follow-up.

We used a Kaplan-Meier analysis to estimate progression-free and overall survival, and treatment comparisons were made with the use of a stratified log-rank test. Post hoc subgroup analy-
Figure 1 (facing page). Progression-free Survival and Subgroup Analyses.

Panel A shows Kaplan-Meier estimates of progression-free survival in the intention-to-treat population. Panel B shows hazard ratios and $95 \%$ confidence intervals for progression-free survival in subgroup analyses, according to baseline characteristics. One patient in each study group underwent randomization in error, so the number of patients in each category may not total the number in the intention-to-treat population. Panel C shows Kaplan-Meier estimates of progression-free survival for patients with an elevated lactate dehydrogenase level at baseline. The vertical lines indicate censoring of data. Eastern Cooperative Oncology Group (ECOG) performance status ranges from 0 to 5 , with higher numbers indicating increasing impairment in activities of daily living. LDH denotes lactate dehydrogenase, and ULN upper limit of the normal range.

ses were performed with the use of an unstratified log-rank test. Efficacy was determined in all patients in the intention-to-treat population; safety analyses were performed in all patients who received at least one dose of a study drug. Treatment beyond progression was defined as the receipt of a study drug more than 15 days after radiologic progression, as defined by RECIST.

\section{RESULTS}

\section{PATIENTS}

Of 947 patients who were screened, 423 patients were randomly assigned to receive either dabrafenib plus trametinib (211 patients) or dabrafenib plus placebo (212 patients) (Table 1, and Fig. $\mathrm{S} 1$ in the Supplementary Appendix). The lack of a BRAF V600E or V600K mutation was the most common reason for exclusion (in 245 patients). Baseline characteristics were similar in the two study groups (Table 1). The median follow-up time was 9 months (range, 0 to 16). Details of patient status and follow-up are provided in Figure $S 1$ in the Supplementary Appendix.

\section{EFFICACY}

In the intention-to-treat population, the estimated median progression-free survival (the primary end point) was longer in the dabrafenib-trametinib group than in the dabrafenib-only group (9.3 months vs. 8.8 months); the hazard ratio for disease progression or death was $0.75(95 \% \mathrm{CI}$, 0.57 to $0.99 ; \mathrm{P}=0.03$ ) (Fig. $1 \mathrm{~A}$ ). The progression- 


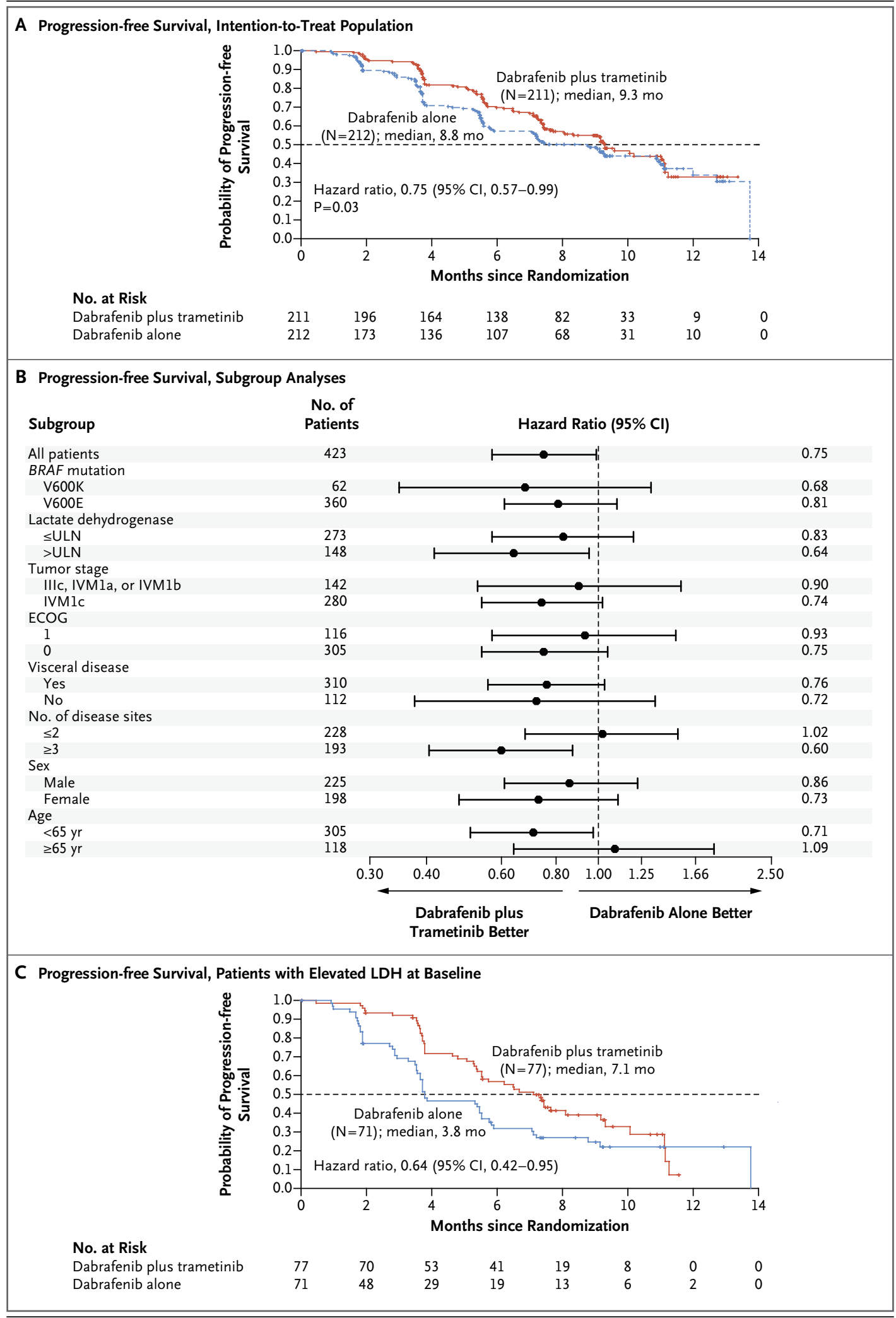

N ENGLJ MED 371;20 NEJM.ORG NOVEMBER 13, 2014

The New England Journal of Medicine 


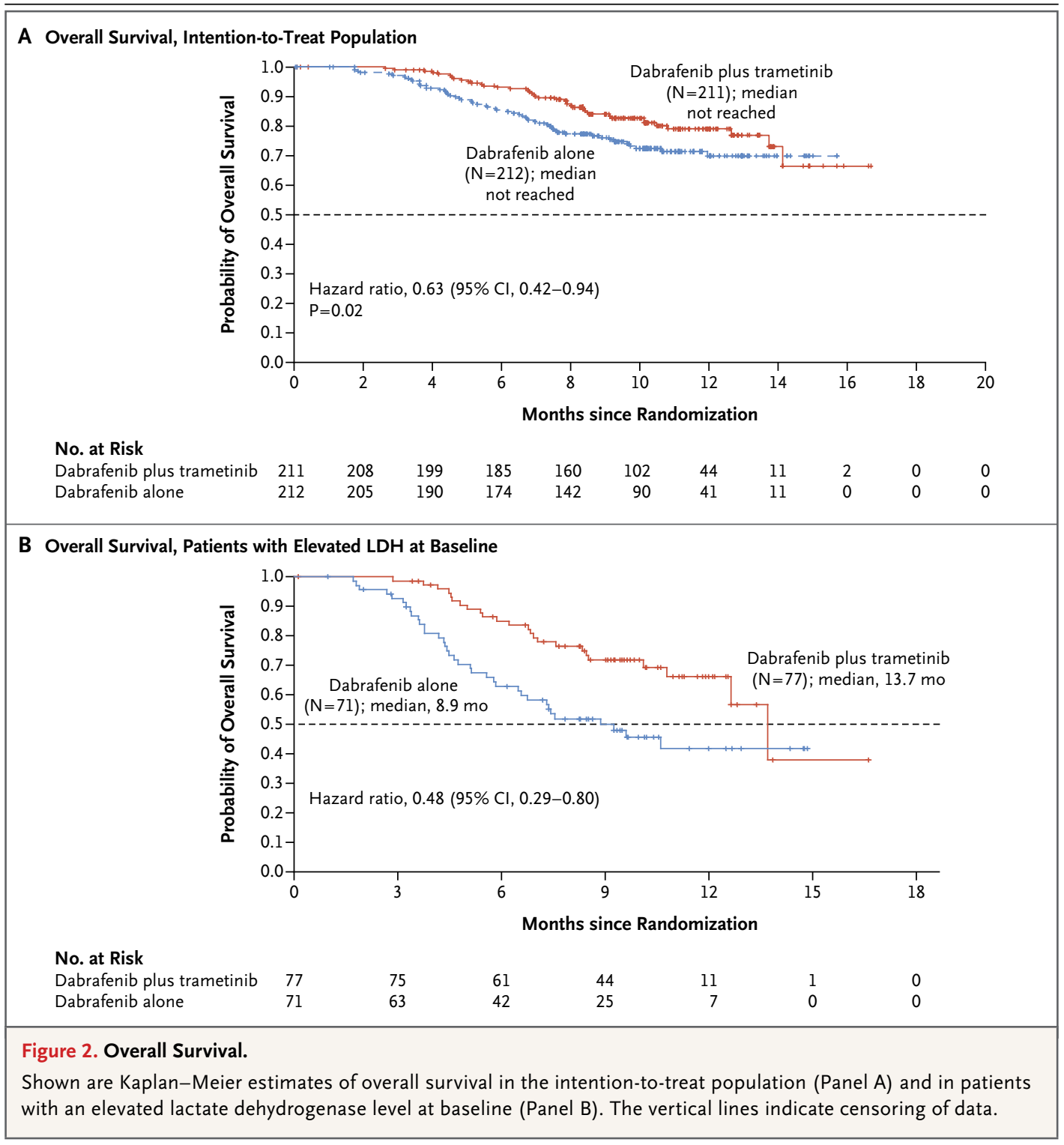

free survival benefit favored the dabrafenib-trametinib group in most of the subgroups that were analyzed (Fig. 1B).

In patients with an elevated lactate dehydrogenase level, the median progression-free survival was 7.1 months in the dabrafenib-trametinib group as compared with 3.8 months in the dabrafenib-only group (hazard ratio for disease progression or death, $0.64 ; 95 \%$ CI, 0.42 to 0.95 ), with 49 events in each group (64\% vs. 69\%) (Fig. 1C). Among patients in the two study groups, there were fewer events in the subgroup with a normal lactate dehydrogenase level: 53 events
$(40 \%)$ in the dabrafenib-trametinib group versus 60 events (43\%) in the dabrafenib-only group (hazard ratio, 0.83 ; $95 \% \mathrm{CI}, 0.57$ to 1.20 ) (Fig. S2A in the Supplementary Appendix).

Within the first 2 months after randomization, data for 6 patients in the dabrafenib-trametinib group and 18 patients in the dabrafenib-only group were censored in the progression-free survival analysis (for details, see the Results section in the Supplementary Appendix). Of the $18 \mathrm{pa}-$ tients in the dabrafenib-only group for whom data were censored, 13 had disease progression on the basis of clinical indications (without radio- 


\begin{tabular}{|c|c|c|c|c|c|c|}
\hline \multirow[t]{2}{*}{ Variable } & \multicolumn{2}{|c|}{ BRAF V600E or V600K } & \multicolumn{2}{|c|}{ BRAF V600E } & \multicolumn{2}{|c|}{ BRAF V600K } \\
\hline & $\begin{array}{l}\text { Dabrafenib } \\
\text { plus } \\
\text { Trametinib } \\
(\mathrm{N}=210)\end{array}$ & $\begin{array}{c}\text { Dabrafenib } \\
\text { Alone } \\
(\mathrm{N}=210)\end{array}$ & $\begin{array}{c}\text { Dabrafenib } \\
\text { plus } \\
\text { Trametinib } \\
(\mathrm{N}=179)\end{array}$ & $\begin{array}{c}\text { Dabrafenib } \\
\text { Alone } \\
(\mathrm{N}=180)\end{array}$ & $\begin{array}{c}\text { Dabrafenib } \\
\text { plus } \\
\text { Trametinib } \\
(\mathrm{N}=31)\end{array}$ & $\begin{array}{c}\text { Dabrafenib } \\
\text { Alone } \\
(\mathrm{N}=30)\end{array}$ \\
\hline \multicolumn{7}{|l|}{ Best response - no. (\%) } \\
\hline Complete response & $22(10)$ & $18(9)$ & $19(11)$ & $16(9)$ & $3(10)$ & $2(7)$ \\
\hline Partial response & $118(56)$ & $90(43)$ & $102(57)$ & $80(44)$ & $16(52)$ & $10(33)$ \\
\hline Stable disease & $54(26)$ & $69(33)$ & $46(26)$ & $62(34)$ & $8(26)$ & $7(23)$ \\
\hline Progressive disease & $13(6)$ & $19(9)$ & $10(6)$ & $11(6)$ & $3(10)$ & $8(27)$ \\
\hline Could not be evaluated & $3(1)$ & $14(7)$ & $2(1)$ & $11(6)$ & $1(3)$ & $3(10)$ \\
\hline \multicolumn{7}{|l|}{ Complete or partial response $†$} \\
\hline No. of patients with response & 140 & 108 & 121 & 96 & 19 & 12 \\
\hline Percentage $(95 \% \mathrm{Cl})$ & $67(60-73)$ & $51(45-58)$ & $68(60-74)$ & $53(46-61)$ & $61(42-78)$ & $40(23-59)$ \\
\hline
\end{tabular}

* Included in this analysis were the 210 patients in each study group who had measurable disease at baseline.

T The absolute difference in the rate of complete or partial response in the group receiving dabrafenib plus trametinib, as compared with the dabrafenib-only group, was 16 percentage points $(95 \% \mathrm{Cl}, 6$ to $25 ; \mathrm{P}=0.002)$ in patients with either $B R A F$ mutation, 15 percentage points $(95 \% \mathrm{Cl}, 4$ to $24 ; \mathrm{P}=0.006)$ in those with the BRAF V600E mutation, and 21 percentage points $(95 \% \mathrm{Cl},-3$ to $46 ; \mathrm{P}=0.10)$ in those with the $B R A F \mathrm{~V} 600 \mathrm{~K}$ mutation. $\mathrm{P}$ values were calculated by means of the chi-square test.

logic confirmation), as determined by the investigator, or had started a new anticancer therapy. In preplanned sensitivity analyses, when clinical progression or initiation of a new anticancer therapy was considered as an event, the hazard ratio for progression and the median progression-free survival for the dabrafenib-trametinib group remained stable (i.e., the median remained the same when clinical progression was considered or decreased by 0.1 month when the initiation of a new anticancer therapy was considered). In contrast, the median progression-free survival in the dabrafenib-only group decreased by 1.2 months when clinical progression was considered and by 1.6 months when the initiation of a new anticancer therapy was considered (Table S1 in the Supplementary Appendix).

At the time of the interim survival analysis, 40 patients $(19 \%)$ in the dabrafenib-trametinib group and 55 patients $(26 \%)$ in the dabrafenibonly group had died (hazard ratio for death, 0.63 ; $95 \% \mathrm{CI}, 0.42$ to $0.94 ; \mathrm{P}=0.02$ ). At 6 months, the overall survival rate was $93 \%$ in the dabrafenib-trametinib group and $85 \%$ in the dabrafenib-only group. However, the between-group difference did not cross the prespecified stopping boundary (two-sided $\mathrm{P}=0.00028$ ). At the time of this report, neither group had reached a median overall survival (Fig. 2A). Patients with an elevated lactate dehydrogenase level appeared to derive a greater survival benefit from combination therapy over monotherapy, with deaths of 24 of 77 patients (31\%) and 36 of 71 patients (51\%), respectively. The median survival among patients with an elevated lactate dehydrogenase level receiving combination therapy was 13.7 months versus 8.9 months among those receiving dabrafenib alone (hazard ratio for death, 0.48; $95 \%$ CI, 0.29 to 0.80 ) (Fig. 2B). In contrast, among patients with a normal lactate dehydrogenase level, deaths were reported in only 16 of 133 patients (12\%) receiving combination therapy and 19 of 140 patients (14\%) receiving dabrafenib alone (Fig. S2B in the Supplementary Appendix).

\section{RESPONSE RATE}

The overall response rate as assessed by investigators was $67 \%$ (95\% CI, 60 to 73 ) in the dabrafenib-trametinib group versus $51 \%(95 \% \mathrm{CI}$, 45 to 58$)$ in the dabrafenib-only group $(\mathrm{P}=0.002)$ (Table 2). In the dabrafenib-trametinib group, 22 patients $(10 \%)$ had a complete response, and 118 (56\%) had a partial response. In the dabrafenib-only group, 18 ( $9 \%)$ had a complete re- 
sponse, and 90 (43\%) had a partial response. The median duration of response was 9.2 months in the dabrafenib-trametinib group and 10.2 months in the dabrafenib-only group on the basis of data that were highly censored because the majority of investigator-assessed responses $(60 \%)$ were still ongoing. Within each of the two study groups, the response rates were slightly lower in the BRAF V600K subgroup than in the predominant V600E subgroup (Table 2).

\section{OTHER ANTICANCER THERAPIES}

Fewer patients in the dabrafenib-trametinib group than in the dabrafenib-only group received a second type of anticancer therapy (43 [20\%] vs. 65 [31\%]) (Table S2 in the Supplementary Appendix). In contrast, more patients in the dabrafenibtrametinib group than in the dabrafenib-only group continued treatment beyond progression (41 [19\%]) vs. $34[16 \%])$, including at the time of data cutoff (19 [46\%] vs. 7 [21\%]). Ipilimumab was the most common subsequent anticancer

\begin{tabular}{|c|c|c|c|c|}
\hline \multirow[t]{3}{*}{ Event } & \multicolumn{2}{|c|}{$\begin{array}{l}\text { Dabrafenib plus Trametinib } \\
\qquad(\mathrm{N}=209)\end{array}$} & \multicolumn{2}{|c|}{$\begin{array}{l}\text { Dabrafenib Alone } \\
\quad(N=211)\end{array}$} \\
\hline & Any Grade'† & Grade 3 & Any Grade† $†$ & Grade 3 \\
\hline & \multicolumn{4}{|c|}{ number of patients (percent) } \\
\hline Any adverse event & $199(95)$ & $66(32)$ & $203(96)$ & $72(34)$ \\
\hline Pyrexiał & $107(51)$ & $12(6)$ & $59(28)$ & $4(2)$ \\
\hline Fatigue & $74(35)$ & $4(2)$ & $74(35)$ & $2(1)$ \\
\hline Headache & $63(30)$ & $1(<1)$ & $62(29)$ & $3(1)$ \\
\hline Nausea & $63(30)$ & 0 & $54(26)$ & $3(1)$ \\
\hline Chills & $62(30)$ & 0 & $33(16)$ & 0 \\
\hline Arthralgia & $51(24)$ & $1(<1)$ & $58(27)$ & 0 \\
\hline Diarrhea & $51(24)$ & $2(1)$ & $30(14)$ & $2(1)$ \\
\hline Rash & $48(23)$ & 0 & $46(22)$ & $2(1)$ \\
\hline Hypertension & $46(22)$ & $8(4)$ & $29(14)$ & $10(5)$ \\
\hline Vomiting & $42(20)$ & $2(1)$ & $29(14)$ & $1(<1)$ \\
\hline Cough & $34(16)$ & 0 & 35 (17) & 0 \\
\hline Peripheral edema & $30(14)$ & $1(<1)$ & $10(5)$ & $1(<1)$ \\
\hline Pain in a limb & $30(14)$ & $3(1)$ & $33(16)$ & $1(<1)$ \\
\hline Decreased appetite & $23(11)$ & $1(<1)$ & $25(12)$ & $2(1)$ \\
\hline Abdominal pain & $22(11)$ & $2(1)$ & $14(7)$ & $3(1)$ \\
\hline Elevated alanine aminotransferase & $22(11)$ & $4(2)$ & $10(5)$ & $1(<1)$ \\
\hline Elevated aspartate aminotransferase & $22(11)$ & $6(3)$ & $7(3)$ & $1(<1)$ \\
\hline Constipation & $22(11)$ & $1(<1)$ & $18(9)$ & 0 \\
\hline Myalgia & $22(11)$ & $1(<1)$ & $24(11)$ & 0 \\
\hline Asthenia & $20(10)$ & $1(<1)$ & $27(13)$ & $1(<1)$ \\
\hline Dizziness & $20(10)$ & 0 & $12(6)$ & 0 \\
\hline Nasopharyngitis & $20(10)$ & 0 & $15(7)$ & 0 \\
\hline Back pain & $19(9)$ & $2(1)$ & $30(14)$ & $4(2)$ \\
\hline Dry skin & $19(9)$ & 0 & $28(13)$ & 0 \\
\hline Pruritus & $17(8)$ & 0 & $26(12)$ & 0 \\
\hline Alopecia & $15(7)$ & 0 & $55(26)$ & 0 \\
\hline Hand-foot syndrome $\mathbb{}$ & $10(5)$ & 0 & $58(27)$ & $1(<1)$ \\
\hline Hyperkeratosis & $7(3)$ & 0 & $68(32)$ & $1(<1)$ \\
\hline Skin papilloma & $3(1)$ & 0 & $45(21)$ & 0 \\
\hline
\end{tabular}




\begin{tabular}{|c|c|c|c|c|}
\hline \multirow[t]{3}{*}{ Event } & \multicolumn{2}{|c|}{$\begin{array}{l}\text { Dabrafenib plus Trametinib } \\
\qquad(\mathrm{N}=\mathbf{2 0 9})\end{array}$} & \multicolumn{2}{|c|}{$\begin{array}{l}\text { Dabrafenib Alone } \\
\qquad(N=211)\end{array}$} \\
\hline & Any Grade $†$ & Grade 3 & Any Grade† & Grade 3 \\
\hline & \multicolumn{4}{|c|}{ number of patients (percent) } \\
\hline \multicolumn{5}{|c|}{ Adverse event of interest occurring in $<10 \%$ of patients } \\
\hline $\begin{array}{l}\text { Cutaneous squamous-cell carcinoma } \\
\text { including keratoacanthoma }\end{array}$ & $5(2)$ & $4(2)$ & $20(9)$ & $8(4)$ \\
\hline Decreased ejection fraction & $9(4)$ & $1(<1)$ & $5(2)$ & $1(<1)$ \\
\hline Chorioretinopathy & $1(<1)$ & 0 & $1(<1)$ & 0 \\
\hline Blurred vision & $5(2)$ & 0 & $4(2)$ & 0 \\
\hline Dermatitis acneiform & $16(8)$ & 0 & $7(3)$ & 0 \\
\hline \multicolumn{5}{|c|}{$\begin{array}{l}\text { Listed are adverse events that occurred in at least } 10 \% \text { of patients who received at least one dose of a study drug in any } \\
\text { group, except as indicated. } \\
\text { A total of eight grade } 4 \text { events occurred in seven patients }(3 \%) \text { in the dabrafenib-trametinib group (anemia, decreased } \\
\text { lymphocyte count, hypoglycemia, pulmonary embolism, brain edema, hepatic hematoma, metastases to central nervous } \\
\text { system, and pancytopenia) and in seven patients }(3 \%) \text { in the dabrafenib-only group (dyspnea, thrombocytopenia, hypo- } \\
\text { kalemia, cutaneous squamous-cell carcinoma, brain edema, hypercalcemia, febrile neutropenia, and hypovolemic shock). } \\
\text { Grade } 5 \text { events were reported in four patients }(2 \%) \text { in the dabrafenib-trametinib group (pneumonia and cerebral hem- } \\
\text { orrhage [in three patients]). } \\
\text { Pyrexia was defined as a body temperature of } 38.5^{\circ} \mathrm{C} \text { or higher. } \\
\text { The hand-foot syndrome included the terms palmar-plantar erythrodysesthesia, palmar-plantar hyperkeratosis, and } \\
\text { palmoplantar keratoderma. }\end{array}$} \\
\hline
\end{tabular}

therapy in the two groups; however, fewer patients in the dabrafenib-trametinib group than in the dabrafenib-only group received ipilimumab (19 [9\%] vs. 32 [15\%]). A similar number of patients in the two groups received subsequent therapy with vemurafenib: $12(6 \%)$ in the dabrafenib-trametinib group and $13(6 \%)$ in the dabrafenib-only group. No patients received monoclonal antibodies targeting programmed death 1 and programmed death ligand 1 .

\section{ADVERSE EVENTS}

Among patients who received at least one dose of a study drug, permanent discontinuations were reported in 19 of 209 patients $(9 \%)$ in the dabrafenib-trametinib group and 11 of 211 patients (5\%) in the dabrafenib-only group. In addition, dose reductions were required in 52 patients (25\%) and 28 patients (13\%), respectively, and interruption of a study drug because of adverse events occurred in 103 patients (49\%) and 70 patients (33\%), respectively. In both the dabrafenibtrametinib group and the dabrafenib-only group, pyrexia was the most common reason for dose interruptions (in 67 patients [32\%] and 28 patients [13\%]) and dose reductions (in 27 patients [13\%] and 6 patients [3\%]). The most common reason for permanent discontinuation was pyrexia in the dabrafenib-trametinib group (in 5 patients [2\%]) and a decreased ejection fraction in the dabrafenib-only group (in 3 patients [1\%]).

The most common adverse events in the two study groups were pyrexia, fatigue, nausea, headache, chills, diarrhea, arthralgia, rash, and hypertension (Table 3). Fewer patients in the dabrafenib-trametinib group than in the dabrafenib-only group had incident cutaneous squamous-cell carcinoma ( $2 \%$ vs. $9 \%$ ) or cutaneous hyperkeratoses (3\% vs. 32\%). Papillomas, alopecia, and the hand-foot syndrome were also less common in the dabrafenib-trametinib group. However, pyrexia, hypertension, peripheral edema, and diarrhea were more common in the dabrafenib-trametinib group than in the dabrafenibonly group (proportion of patients with pyrexia, $51 \%$ vs. $28 \%$ ) (Table 3, and Fig. S3 in the Supplementary Appendix). There were no cases of retinal-vein occlusion. Of the 107 patients in the dabrafenib-trametinib group who had pyrexia, 67 (63\%) had a second episode, and 50 (47\%) had three or more episodes. The median time to the onset of the first episode of pyrexia was 4.3 weeks, and the median duration was 3 days. During an acute episode of pyrexia, $63 \%$ of pa- 
tients had a dose interruption and $25 \%$ had a dose reduction, with resolution of pyrexia in $97 \%$ of the patients. Glucocorticoids were used for 3 weeks or more as prophylaxis for pyrexia in 30 patients in the dabrafenib-trametinib group after the first or second event. Pharmacokinetic analysis showed a possible association between pyrexia and exposure to the hydroxy-dabrafenib metabolite and, to a lesser extent, to dabrafenib in the two study groups. Similarly, trametinib exposure was associated with pyrexia in the combination group (see the Methods and Results sections and Fig. S4 and S5 in the Supplementary Appendix).

Grade 3 or 4 adverse events occurred in 73 patients $(35 \%)$ in the dabrafenib-trametinib group and 79 patients (37\%) in the dabrafenib-only group. Noncutaneous cancers and new primary melanomas were uncommon (Table S3 in the Supplementary Appendix). Four deaths related to adverse events occurred in the dabrafenibtrametinib group: three from cerebral hemorrhage (two during receipt of study treatment and one 5 days after cessation) and one from pneumonia (22 days after cessation of treatment). All four events were considered by the investigator to be unrelated to study treatment. No deaths related to adverse events occurred in the dabrafenib-only group (Table S3 in the Supplementary Appendix).

\section{DISCUSSION}

In this phase 3 study, we found a significant $25 \%$ relative reduction in the risk of disease progression among patients with metastatic melanoma with BRAF V600E or V600K mutations who received first-line treatment with a combination of dabrafenib and trametinib, as compared with dabrafenib alone. The combination therapy, as compared with monotherapy, was associated with a $37 \%$ relative reduction in the risk of death at the interim survival analysis, but this result did not cross the prespecified stopping boundary. Notably, this difference in survival was observed despite the increased use of new therapies after the discontinuation of monotherapy, including therapies with a proven survival benefit (e.g., ipilimumab). ${ }^{20,21}$ The overall response rate was also significantly higher in the group receiving both dabrafenib and trametinib. Resistance to singleagent BRAF inhibition is acquired predominantly through reactivation of the MAPK pathway. ${ }^{5-7,22-26}$ Our study shows that the inhibition of the MAPK pathway at two nodes rather than one decreases the risk of progression (and therefore delays resistance) by $25 \%$.

In a post hoc subgroup analysis, we found that patients with poorer prognostic features appeared to benefit more from a combination of dabrafenib and trametinib than did those in the overall study population. At the time of the interim survival analysis, patients with an elevated lactate dehydrogenase level who received the combination therapy, as compared with monotherapy, had a $52 \%$ relative reduction in the risk of death (hazard ratio, 0.48). Since events tend to occur earlier in patients with poorer prognostic features, longer follow-up will be required to assess the effects of combination therapy as compared with monotherapy in patients with better prognostic features, such as a normal lactate dehydrogenase level or stage M1a or M1b melanoma (i.e., only distant skin, subcutaneous, or nodal metastases or lung metastases).

Our study used a control regimen that had a proven survival benefit. ${ }^{2}$ It had a double-blind design and did not allow crossover at the time of progression. In addition, the median progression-free survival of 8.8 months in the dabrafenib-only group was longer than that in previous trials of dabrafenib and vemurafenib in which the median progression-free survival was 5.5 to 6.9 months. ${ }^{1-4,17,27-29}$ The long plateau at the median progression-free survival point in the dabrafenib-only group may account in part for its increased value in our study (Fig. 1A). In addition, the preplanned sensitivity analysis showed that the median progression-free survival for dabrafenib was unstable. Data for patients who had clinical progression or received a new anticancer therapy without radiographic evidence of progression were censored (which occurred more frequently in the dabrafenib-only group than in the combination-therapy group in the first 2 months of the study). Thus, the median progression-free survival for the dabrafenib group decreased from 8.8 months to 7.6 months when clinical progression was included as an event and decreased from 8.8 months to 7.2 months when receipt of a new anticancer therapy was included.

Patients receiving the combination therapy required more dose modifications than did those 
receiving monotherapy. There was no significant between-group difference in the frequency of adverse events, including grade 3 and 4 toxic effects. However, the types of toxic effects differed. Hyperproliferative cutaneous events, including cutaneous squamous-cell carcinoma and other events related to paradoxical activation of the MAPK pathway, which show the oncogenic potential of BRAF inhibitors, ${ }^{11-13}$ were significantly abrogated by the addition of the MEK inhibitor trametinib. ${ }^{12}$ There were no deaths in either study group that were considered by the investigators to be related to treatment.

Pyrexia occurred more frequently in the combination group than in the monotherapy group and was the most common reason for treatment discontinuation. Although there was an apparent relationship between pyrexia and exposure to the hydroxy-dabrafenib metabolite and trametinib in this study, the cause of pyrexia remains unknown. Pyrexia has been shown to respond well to immediate temporary cessation of dabrafenib or both dabrafenib and trametinib, which were restarted once the patient was well and afebrile for at least 24 hours; prophylactic glucocorticoids may prevent recurrent episodes..$^{30}$ The education of patients regarding pyrexia and the need to interrupt treatment at the first sign of this side effect is critical to ensure that the episode is not prolonged or complicated by dehydration or hypotension.

In conclusion, in our study involving previously untreated patients who had metastatic melanoma with BRAF V600E or V600EK mutations, the combination of dabrafenib and trametinib, as compared with dabrafenib alone, reduced the risk of progression by $25 \%$ and improved the response rate. At the time of the interim analysis, overall survival was longer in the combination group, but the prespecified stopping boundary was not crossed.

\section{Supported by GlaxoSmithKline.}

Disclosure forms provided by the authors are available with the full text of this article at NEJM.org.

The authors' full names and academic degrees are as follows: Georgina V. Long, M.D., Ph.D., Daniil Stroyakovskiy, M.D., Helen Gogas, M.D., Evgeny Levchenko, M.D., Filippo de Braud, M.D., James Larkin, M.D., Claus Garbe, M.D., Ph.D., Thomas Jouary, M.D., Axel Hauschild, M.D., Ph.D., Jean Jacques Grob, M.D., Ph.D., Vanna Chiarion Sileni, M.D., Celeste Lebbe, M.D., Mario Mandalà, M.D., Michael Millward, M.D., Ana Arance, M.D., Ph.D., Igor Bondarenko, M.D., Ph.D., John B.A.G. Haanen, M.D., Ph.D., Johan Hansson, M.D., Ph.D., Jochen Utikal, M.D., Ph.D., Virginia Ferraresi, M.D., Nadezhda Kovalenko, M.D., Ph.D., Peter Mohr, M.D., Volodymyr Probachai, M.D., Dirk Schadendorf, M.D., Ph.D., Paul Nathan, M.D., Caroline Robert, M.D., Antoni Ribas, M.D., Ph.D., Douglas J. DeMarini, Ph.D., Jhangir G. Irani, M.A., Michelle Casey, Ph.D., Daniele Ouellet, Ph.D., Anne-Marie Martin, Ph.D., Ngocdiep Le, M.D., Ph.D., Kiran Patel, M.D., and Keith Flaherty, M.D.

The authors' affiliations are as follows: the Melanoma Institute Australia, University of Sydney, Mater Hospital, Sydney (G.V.L.), and Sir Charles Gairdner Hospital, Perth, WA (M. Millward) - both in Australia; Moscow City Oncology Hospital \#62, Moscow (D.S.), Petrov Research Institute of Oncology, Saint Petersburg (E.L.), and Volograd Regional Oncology Dispensary \#3, Volzhsky (N.K.) — all in Russia; University of Athens, Athens (H.G.); Fondazione Istituto di Ricovero e Cura a Carattere Scientifico (IRCCS) Istituto Nazionale dei Tumori, Milan (F.B.), Veneto Oncology Institute-IRCCS, Padua (V.C.S.), Papa Giovanni XXIII Hospital, Bergamo (M. Mandalà), and Regina Elena National Cancer Institute, Rome (V.F.) — all in Italy; Royal Marsden NHS Foundation Trust, London (J.L.), and Mount Vernon Cancer Centre, Northwood (P.N.) - both in the United Kingdom; University of Tübingen, Tübingen (C.G.), University Hospital Schleswig-Holstein, Campus Kiel, Kiel (A.H.), German Cancer Research Center (DKFZ) and University Medical Center Mannheim, Ruprecht-Karl University of Heidelberg, Mannheim and Heidelberg (J.U.), Elbe Kliniken Stade-Buxtehude, Buxtehude (P.M.), and University Hospital Essen, Essen (D.S.) — all in Germany; Hôpital Francois Mitterrand, Pau (T.J.), Hôpital de la Timone, Marseille (J.J.G.), Hôpital Saint-Louis, Paris (C.L.), and Institut Gustave Roussy, Villejuif-Paris (C.R.) — all in France; Hospital Clinic, Barcelona (A.A.); Dnipropetrovsk Medical Academy, Clinical Hospital \#4 (I.B.) and Dnipropetrovsk Clinical Oncology Center of Dnipropetrovsk State Council (V.P.), Dnipropetrovsk, Ukraine; Netherlands Cancer Institute, Amsterdam (J.B.A.G.H.); Karolinska Institutet, Stockholm (J.H.); University of California, Los Angeles, Los Angeles (A.R.); GlaxoSmithKline, Collegeville, PA (D.J.D., J.I., M.C., A.-M.M., N.L., K.P.); GlaxoSmithKline, Research Triangle Park, NC (D.O.); and Massachusetts General Hospital Cancer Center, Boston (K.F.).

\section{REFERENCES}

1. Chapman PB, Hauschild A, Robert C, et al. Improved survival with vemurafenib in melanoma with BRAF V600E mutation. N Engl J Med 2011;364:2507-16.

2. Hauschild A, Grob JJ, Demidov LV, et al. Dabrafenib in BRAF-mutated metastatic melanoma: a multicentre, open-label, phase 3 randomised controlled trial. Lancet 2012;380:358-65.

3. Chapman PB, Hauschild A, Robert C, et al. Updated overall survival (OS) results for BRIM-3, a phase III randomized, open-label, multicenter trial comparing BRAF inhibitor vemurafenib (vem) with dacarbazine (DTIC) in previously untreated patients with BRAFV600E-mutated melanoma. J Clin Oncol 2012;30:Suppl: 8502. abstract.

4. Hauschild A, Grob JJ, Demidov LV, et al. An update on BREAK-3, a phase III, randomized trial: dabrafenib (DAB) versus dacarbazine (DTIC) in patients with
BRAF V600E-positive mutation metastatic melanoma (MM). J Clin Oncol 2013;31: Suppl:9013. abstract.

5. Rizos H, Menzies AM, Pupo GM, et al. BRAF inhibitor resistance mechanisms in metastatic melanoma: spectrum and clinical impact. Clin Cancer Res 2014;20:196577.

6. Van Allen EM, Wagle N, Sucker A, et al. The genetic landscape of clinical resistance to RAF inhibition in metastatic 
melanoma. Cancer Discov 2014;4:94109.

7. Shi H, Hugo W, Kong X, et al. Acquired resistance and clonal evolution in melanoma during BRAF inhibitor therapy. Cancer Discov 2014;4:80-93.

8. Hatzivassiliou G, Song K, Yen I, et al. RAF inhibitors prime wild-type RAF to activate the MAPK pathway and enhance growth. Nature 2010;464:431-5.

9. Poulikakos PI, Zhang C, Bollag G, Shokat KM, Rosen N. RAF inhibitors transactivate RAF dimers and ERK signalling in cells with wild-type BRAF. Nature 2010;464:427-30.

10. Heidorn SJ, Milagre C, Whittaker S, et al. Kinase-dead BRAF and oncogenic RAS cooperate to drive tumor progression through CRAF. Cell 2010;140:209-21.

11. Callahan MK, Rampal R, Harding JJ, et al. Progression of RAS-mutant leukemia during RAF inhibitor treatment. N Engl J Med 2012;367:2316-21.

12. Su F, Viros A, Milagre C, et al. RAS mutations in cutaneous squamous-cell carcinomas in patients treated with BRAF inhibitors. N Engl J Med 2012;366:207-15. 13. Oberholzer PA, Kee D, Dziunycz P, et al. RAS mutations are associated with the development of cutaneous squamous cell tumors in patients treated with RAF inhibitors. J Clin Oncol 2012;30:316-21.

14. Flaherty KT, Robert C, Hersey P, et al. Improved survival with MEK inhibition in BRAF-mutated melanoma. N Engl J Med 2012;367:107-14.

15. Paraiso KH, Fedorenko IV, Cantini LP, et al. Recovery of phospho-ERK activity allows melanoma cells to escape from BRAF inhibitor therapy. Br J Cancer 2010; 102:1724-30.

16. Infante JR, Falchook GS, Lawrence DP, et al. Phase I/II study to assess safety, pharmacokinetics, and efficacy of the oral MEK 1/2 inhibitor GSK1120212 (GSK212) dosed in combination with the oral BRAF inhibitor GSK2118436 (GSK436). J Clin Oncol 2011;29:Suppl:8503. abstract. 17. Flaherty KT, Infante JR, Daud A, et al Combined BRAF and MEK inhibition in melanoma with BRAF V600 mutations. N Engl J Med 2012;367:1694-703.

18. Eisenhauer EA, Therasse P, Bogaerts J, et al. New response evaluation criteria in solid tumours: revised RECIST guideline (version 1.1). Eur J Cancer 2009;45:228-47. 19. National Cancer Institute. Common Terminology Criteria for Adverse Events (CTCAE), version 4.0. May 29, 2009 (http:// ctep.cancer.gov/protocolDevelopment/ electronic_applications/ctc.htm).

20. Hodi FS, O'Day SJ, McDermott DF, et al. Improved survival with ipilimumab in patients with metastatic melanoma. N Engl J Med 2010;363:711-23. [Erratum, N Engl J Med 2010;363:1290.]

21. Robert C, Thomas L, Bondarenko I, et al. Ipilimumab plus dacarbazine for previously untreated metastatic melanoma. N Engl J Med 2011;364:2517-26.

22. Shi H, Moriceau G, Kong $X$, et al. Melanoma whole-exome sequencing identifies (V600E)B-RAF amplification-mediated acquired B-RAF inhibitor resistance. Nat Commun 2012;3:724.

23. Nazarian R, Shi H, Wang Q, et al. Mela- nomas acquire resistance to $\mathrm{B}-\mathrm{RAF}(\mathrm{V} 600 \mathrm{E})$ inhibition by RTK or N-RAS upregulation. Nature 2010;468:973-7.

24. Poulikakos PI, Persaud Y, Janakiraman $\mathrm{M}$, et al. RAF inhibitor resistance is mediated by dimerization of aberrantly spliced BRAF(V600E). Nature 2011;480: 387-90.

25. Wagle N, Emery C, Berger MF, et al. Dissecting therapeutic resistance to RAF inhibition in melanoma by tumor genomic profiling. J Clin Oncol 2011;29:3085-96. 26. Villanueva J, Vultur A, Lee JT, et al. Acquired resistance to BRAF inhibitors mediated by a RAF kinase switch in melanoma can be overcome by cotargeting MEK and IGF-1R/PI3K. Cancer Cell 2010; 18:683-95.

27. Sosman JA, Kim KB, Schuchter L, et al. Survival in BRAF V600-mutant advanced melanoma treated with vemurafenib. N Engl J Med 2012;366:707-14.

28. Falchook GS, Long GV, Kurzrock R, et al. Dabrafenib in patients with melanoma, untreated brain metastases, and other solid tumours: a phase 1 dose-escalation trial. Lancet 2012;379:1893-901.

29. Ascierto PA, Minor D, Ribas A, et al. Phase II trial (BREAK-2) of the BRAF inhibitor dabrafenib (GSK2118436) in patients with metastatic melanoma. J Clin Oncol 2013;31:3205-11.

30. Lee CI, Menzies AM, Haydu LE, et al. Features and management of pyrexia with combined dabrafenib and trametinib in metastatic melanoma. Melanoma Res 2014; 24:468-74.

Copyright (c) 2014 Massachusetts Medical Society. 